\title{
STRONGLY MIXING TRANSFORMATIONS AND GEOMETRIC DIAMETERS
}

\author{
HUSE FATKIĆ AND MEHMED BRKIĆ \\ Dedicated to Professor Mustafa Kulenović on the occasion of his 60th birthday
}

\begin{abstract}
In this paper, we investigate metric properties and dispersive effects of strongly mixing transformations on general metric spaces endowed with a finite measure; in particular, we investigate their connections with the theory of generalized (geometric) diameters on general metric spaces. We first show that the known result by Rice $[17$, Theorem 2] (motivated by some physical phenomena and offer some clarifications of these phenomena), which is a substantial improvement of Theorems 1 and 2 due to Erber, Schweizer and Sklar [4], can be generalized in such a way that this result remains valid when "ordinary diameter" is replaced by "geometric diameter of any finite order". Next we show that "ordinary essential diameter" in the mentioned Rice's result can be replaced by "essential geometric diameter of any finite order". These results also complement the previous results of Fatkić [ 6,8,10], Saff [18] and Sempi [20].
\end{abstract}

\section{INTRODUCTION AND PRELIMINARIES}

In the broadest sense abstract dynamical systems and ergodic theory is the study of the qualitative properties of actions of groups on spaces (e.g. measure spaces, or topological spaces, or smooth manifolds). In this work we shall study actions of the group $\mathbf{Z}$ of integers on a measure space $X$, i.e., we study a transformation $T: X \rightarrow X$ and its iterates $T^{n}, n \in \mathbf{Z}$.

It is customary in abstract dynamical systems and ergodic theory to assume that the underlying space is either a finite or $\sigma$-finite measure space. We shall assume that the measure is finite. Specifically, we shall investigate metric properties and dispersive effects of strongly mixing transformations on general metric spaces endowed with a finite measure; in particular, we

2000 Mathematics Subject Classification. 37A25, 26A18, 28A10.

Key words and phrases. Abstract dynamical system, essential diameter, geometric diameter of finite order, strongly mixing dynamical system; strongly mixing transformation, weakly mixing transformation. 
investigate some connections between the theory of mixing dynamical systems with discrete time and the theory of generalized (geometric) diameters on metric spaces. We shall generally refer to Billingsley [1], Cornfeld, Fomin and Sinai [3], Fatkić [7], Hille [14], Schweizer and Sklar [19] and Walters [22].

We use $\mathbf{N}$ to denote the set of natural numbers, $\mathbf{N}_{0}$ to denote the set of nonnegative integers, $\mathbf{Z}$ to denote the set of integers, $\mathbf{R}$ to denote the set of real numbers (the reals) and $\overline{\mathbf{R}}$ to denote the extended reals, i.e., the set of real numbers with the symbols $-\infty,+\infty$ adjoinded, and ordered via $-\infty<x<+\infty$ for all real $x$. Thus $\overline{\mathbf{R}}$ is a closed interval. The empty set will be denoted by $\varnothing$.

Suppose $(X, \mathscr{A}, \mu)$ is a finite measure space. As usual, a transformation $T: X \rightarrow X$ is called:

(i) measurable $(\mu-$ measurable $)$ if, for any $A$ in $\mathscr{A}$, the inverse image $T^{-1}(A)$ is in $\mathscr{A}$

(ii) measure-preserving if $T$ is measurable and $\mu\left(T^{-1}(A)\right)=\mu(A)$ for any $A$ in $\mathscr{A}$ (or, equivalently, measure $\mu$ is said to be invariant under $T$ );

(iii) ergodic if the only members $A$ of $\mathscr{A}$ with $T^{-1}(A)=A$ satisfy $\mu(A)=$ 0 or $\mu(X \backslash A)=0$;

(iv) weakly mixing (or weak-mixing) (with respect to $\mu$ ) if $T$ is $\mu$ measurable and

$$
\lim _{n \rightarrow \infty} \frac{1}{n} \sum_{i=0}^{n-1}\left|\mu\left(T^{-i}(A) \cap B\right)-\frac{\mu(A) \mu(B)}{\mu(X)}\right|=0
$$

for any two $\mu$-measurable subsets $A, B$ of $X$;

(v) strongly mixing (or mixing, strong-mixing) (with respect to $\mu$ ) if $T$ is $\mu$-measurable and

$$
\lim _{n \rightarrow \infty} \mu\left(T^{-n}(A) \cap B\right)=\frac{\mu(A) \mu(B)}{\mu(X)}
$$

for any two $\mu$-measurable subsets $A, B$ of $X$.

We say that the transformation $T: X \rightarrow X$ is invertible if $T$ is one-to-one (monic) and such that $T(A)$ is $\mu$-measurable whenever $A$ is $\mu$-measurable subset of $X$.

A transformation $T$ on a finite measure space $(X, \mathscr{A}, \mu)$ is said to be measurability - preserving if $T(\mathscr{A}) \subseteq \mathscr{A}$ (i.e., if $T(A)$ is $\mu$ - measurable whenever $A$ is $\mu$-measurable ([9, Definition 1$])$. In this case we also say that the transformation $T$ preserves $\mu$ - measurability.

The objects of interest are not really measure-preserving transformations, as is well-known, but equivalence classes of such transformations; two transformations are equivalent if they differ only on a set of measure zero. 
Measure-preserving transformations arise, e.g., in the investigation of classical dynamical systems. In this case $T$ is first obtained as a continuous transformation of some compact topological space, and the existence of an invariant measure $\mu$ is proved. The system $(X, \mathscr{A}, \mu, T)$ is then abstracted from the topological setting. Therefore, if $(X, \mathscr{A}, \mu)$ is a finite measure space, and $T: X \rightarrow X$ is a measure-preserving transformation (with respect to $\mu$ ) , then we say that $\Phi:=(X, \mathscr{A}, \mu, T)$ is an abstract dynamical system. An abstract dynamical system is often called a dynamical system with discrete time or a measure-theoretic dynamical system or an endomorphism. We shall say that the abstract dynamical system $\Phi$ is: (i) invertible if $T$ is invertible; (ii) ergodic if $T$ is ergodic; (iii) weakly (resp. strongly) mixing if $T$ is weakly (resp. strongly) mixing (see [3, pp. 6 - 26]; but see also [2], [5], [6-8], [10], [15-17] and [19-22]). For example, in [2], the global invariant properties of a class of exactly solvable area-preserving mixing transformations of the two-dimensional torus are carefully analyzed. Starting from the closed-form solution of the expanding subbundle, a nonuniform stationary measure $\mu_{w}$ is derived analytically, providing a concrete example for which the connections between geometric and measure-theoretic approaches to chaotic dynamics can be worked out explicitly. The implications of the results for physically realizable mixing systems are also considered.

If $T$ is a strongly mixing transformation of a finite measure space $(X$, $\mathscr{A}, \mu)$, then, as is well-known, $T$ is both measure-preserving and ergodic. Furthermore, if $T: X \rightarrow X$, in addition (to being strongly mixing on $X$ with respect to $\mu$ ), is invertible, then (1.1) is equivalent to (the well-known result):

$$
\lim _{n \rightarrow \infty} \mu\left(T^{n}(A) \cap B\right)=\frac{\mu(A) \mu(B)}{\mu(X)}
$$

for any $\mu$ - measurable subsets $A, B$ of $X$.

Let us now give an example of a strongly mixing transformation which is not invertible.

Example 1.1. (see [8, p. 50]) Let $A$ consist of the Borel subsets of the half-open unit interval $X:=[0,1)$, with Lebesgue measure for $\mu$ and let $T(x)=2 x(\bmod 1)$ on $[0,1) . T$ is called a dyadic transformation (or a (angle) doubling map, Bernoulli map, bit shift map, dyadic map, $2 x(\bmod 1)$ map or sawtooth map). An application of the well-known criterion which is useful when checking whether or not examples have the strongly mixing properties (see [10, Example 2.1, p.163]) and, e.g., [22, Theorem 1.17]) shows that the dyadic transformation $T$ is strongly mixing. Since $T$ obviously is not oneto-one, it follows that the dyadic transformation is strongly mixing but not invertible. 
In this work we consider a metric space/( extended metric spaces) $(X, d)$ on which a finite measure $\mu$ is defined. The domain of $\mu$, a $\sigma$-algebra $\mathscr{A}$ of subsets of $X$, is assumed to include all Borel sets in $(X, d)$; in particular, therefore, all open balls in $(X, d)$ are $\mu$-measurable.

The (ordinary) diameter of a subset $A$ of $X$, i.e., the supremum of the set $\{d(x, y) \mid x, y \in A\}$, will be denoted by $\operatorname{diam}(A)$. We can define the diameter of the empty set (the case $A=\varnothing$ ) as 0 or $-\infty$, as we like. But, like many other authors, we prefer to treat the empty set as a special case, assigning it a diameter equal to 0 , i.e., $\operatorname{diam}(\varnothing)=0$, which corresponds to taking the codomain of $d$ to be the set of all nonnegative real numbers.

If $A$ is $\mu$-measurable, then the (ordinary) essential diameter of $A$, denoted by ess $\operatorname{diam}(A)$, is the infimum of the set of diameters of all $\mu$-measurable sets $B$ such that $B \subseteq A$ and $\mu(B)=\mu(A)$, i.e.,

ess $\operatorname{diam}(A):=\inf \{\operatorname{diam}(B) \mid B$ is $\mu$-measurable, $B \subseteq A, \mu(B)=\mu(A)\}$.

$\operatorname{Both} \operatorname{diam}(A)$ and ess $\operatorname{diam}(A)$ may be infinite. Note that ess $\operatorname{diam}(A)$ $\leq \operatorname{diam}(A)$ for all $\mu$-measurable subsets $A$ of $X$. If $B \subseteq A$ and both are $\mu$-measurable then ess $\operatorname{diam}(B) \leq \operatorname{ess} \operatorname{diam}(A)$.

Place $k$ points on a compact set $A$ in the complex plane so that they are "as far apart" as possible in the sense of the geometric mean of the pairwise distances between the points. Since the number of different pairs of $k$ points is $k(k-1) / 2$, we consider the quantity $\delta_{k}(A)$, for each integer $k \geq 2$,

$$
\delta_{k}(A):=\max \left\{\left(\sqrt[k]{2} \sqrt{\prod_{1 \leq i<j \leq k}\left|z_{i}-z_{j}\right|}: z_{1}, z_{2}, \ldots, z_{k} \in A\right\},\right.
$$

which is called the geometric diameter of order $k$ of the set $A$ (or the $k$ diameter of $A$ ).

In 1923, M. Fekete (see, e.g.,[14]) showed that the $\operatorname{limit}_{k \rightarrow \infty} \delta_{k}(A)$ exists, and he called it the transfinite diameter of $A$. In 1931, G. Pólya and G. Szegö extended the concept to compact sets in three - dimensional space $\mathbf{R}^{3}$ and showed that the transfinite diameter coincides with the logarithmic capacity. Further generalizations of this concept were made by them and by F. Leja. Finally E. Hille summarized and unified the previous generalizations (see [14]; but for some recent related result, see, e.g., [7], [13] and [18]).

The concepts of geometric diameters and transfinite diameter make sense in a fairly general context. These notions for arbitrary sets in general metric spaces are introduced (as corresponding generalizations of the concept introduced by Fekete) in the following way:

Let $(X, d)$ be a metric space/(extended metric space), and let $A$ be a subset of $X$. For any positive integer $k \geq 2$, we define the the geometric diameter of order $k$ of $A$ ( $k$ th diameter of $A$ ), denoted by $\delta_{k}(A)$, to be the 
quantity

$$
\delta_{k}(A):=\sup \left\{\left(\sqrt[k]{2} \sqrt{\prod_{1 \leq i<j \leq k} d\left(x_{i}, x_{j}\right)} \mid x_{1}, \ldots, x_{k} \in A\right\} .\right.
$$

Note that $\delta_{2}(A)$ is the (ordinary) diameter of the set $A$. The sequence $\left(\delta_{k}(A)\right)$ can be shown to be decreasing (see, e.g., [14] and [18]), and therefore has a limit as $k$ tends to infinity. By definition, the (geometric) transfinite diameter of $A$ is

$$
\tau(A):=\lim _{k \rightarrow \infty} \delta_{k}(A) .
$$

Note that $0 \leq \tau(A) \leq \delta_{k}(A) \leq \operatorname{diam}(A)$, and that $B \subseteq A$ implies $\tau(B) \subseteq$ $\tau(A)$.

Example 1.2. (see [18, p. 167]) Let $A$ be the closed unit disk (or the unit circle). Then

$$
\delta_{k}(A)=\sqrt[k-1]{k}, \quad \tau(A)=1 .
$$

Example 1.3 (see 18, p. 169). The closed set $\{0\} \cup\{1 / k: k=1,2, \ldots\}$ has transfinite diameter zero.

If $A$ is $\mu$-measurable subset of $X$, then, for any positive integer $k \geq 2$, the essential geometric diameter of order $k$ of $A$, denoted by ess $\delta_{k}(A)$, is the infimum of the set of geometric diameters of all $\mu$-measurable sets $B$ such that $B \subseteq A$ and $\mu(B)=\mu(A)$, i.e.,

ess $\delta_{k}(A)=\inf \left\{\delta_{k}(B) \mid B\right.$ is $\mu$-measurable, $\left.B \subseteq A, \mu(B)=\mu(A)\right\}$.

Both $\delta_{k}(A)$ and ess $\delta_{k}(A)$ may be infinite. Note that ess $\delta_{k}(A) \leq \delta_{k}(A)$ for all $\mu$-measurable subsets $A$ of $X$ and for any positive integer $k \geq 2$. If $B \subseteq A$ and both are $\mu$-measurable, then $\delta_{k}(B) \leq \delta_{k}(A)$, ess $\delta_{k}(B) \leq$ ess $\delta_{k}(A) \leq \operatorname{ess} \operatorname{diam}(A)$, for any positive integer $k \geq 2$. Also, for any positive integer $k \geq 2$, we have $\delta_{k}(\varnothing)=0$, ess $\delta_{k}(\varnothing)=0$.

The general theory of geometric diameters and transfinite diameters plays an important role in complex analysis. It is related to the logarithmic potential theory with applications to approximation theory and the Čebyšev constant (see, e.g., [18]).

Remark 1.1 (see 18, p. 169). The transfinite diameter $\tau$ (considered as a set function) has some of the properties of Lebesgue measure on compact subsets in the complex plane; in fact, the transfinite diameter of a line segment of length $L$ is $L / 4$. However, $\tau$ fails to be subadditive; $\tau(A \cup B)$ may exceed the sum $\tau(A)+\tau(B)$.

Investigations in [4 - 12], [16], [17] and [19 - 23] have shown, however, that many important consequences of (1.2) persist in the absence of invertibility 
and/or the strongly mixing property. The following results (the most useful results of these investigations for the goals of this paper) is due to R.E. Rice [17, Theorems 1 and 2]:

Theorem 1.1. (Rice, 1978) Let $T$ be a strongly mixing transformation on the normalized measure space (probability space) $(X, \mathscr{A}, \mu)$. If $T$ is measurability - preserving, then for any $\mu$-measurable subsets $A, B$ of $X$,

$$
\lim _{n \rightarrow \infty} \mu\left(T^{n}(A) \cap B\right)=\mu(B) \lim _{n \rightarrow \infty} \mu\left(T^{n}(A)\right) .
$$

Theorem 1.2. (Rice, 1978) Let $(X, d)$ be a metric space, let $\mathscr{A}$ be a $\sigma$ algebra of subsets of $X$ and $\mu$ a normalized (probability) measure on $\mathscr{A}$. Suppose further that every open ball in $(X, d)$ is $\mu$-measurable and has positive measure. Let $T$ be a transformation on $X$ that is strongly mixing with respect to $\mu$ and suppose that $A$ is $\mu$-measurable subset of $X$ with positive measure. Then

(i)

$$
\lim _{n \rightarrow \infty} \operatorname{diam}\left(T^{n}(A)\right)=\operatorname{diam}(X) .
$$

(ii) If in addition, $T$ is forward measurable, i.e., if $T(B)$ is $\mu$-measurable whenever $B$ is $\mu$-measurable, then

$$
\lim _{n \rightarrow \infty} \operatorname{ess} \operatorname{diam}\left(T^{n}(A)\right)=\operatorname{diam}(X) .
$$

Theorems 1.1. and 1.2 have many consequences which are of interest because of the extreme simplicity of both their mathematical and physical realizations. Among others, these consequences have great relevance in the discussion of the recurrence paradox of Statistical Mechanics (see the previous results of T. Erber, B. Schweizer and A. Sklar [4], B. Schweizer and A. Sklar [19, pp. 181 - 190 and (in Dover Edition) 295 - 297], T. Erber and A. Sklar [5], C. Sempi [20] and H. Fatkić [6 - 8]). It is therefore interesting to investigate how the conclusions of Theorem 1.2 must be modified when the the ordinary diameter is replaced by the geometric diameter of any finite order.

Theorem 1.1 has been applied by H. Fatkić $[7,8]$ obtaining a very general and subtle characterization of measurability-preserving ergodic transformations, and Theorem 1.2 has been extended by C. Sempi [20], H. Fatkić $[6,7,9,10]$, and H. Fatkić and S. Sekulović [11] from strongly mixing transformations to weakly mixing transformations (see also [19 (Dover Edition), p. 297]).

\section{MAIN RESUlts}

Our aim in this work is to show that the known R. E. Rice's Theorem1.2 [17, Theorem 2] (motivated by some physical phenomena and offer some 
clarifications of these phenomena), which is a substantial improvement of Theorems 1 and 2 due to T. Erber, B. Schweizer and A. Sklar [4], can be generalized in such a way that (1.7) remains valid when "(ordinary) diameter" is replaced by "geometric diameter of any finite order" and that (1.8) remains valid when "(ordinary) essential diameter" is replaced by "essential geometric diameter of any finite order". These results also complement the previous results of H. Fatkić [6 - 10], E. B. Saff [18], C. Sempi [20], H. Fatkić and S. Sekulović [11], and H. Fatkić, S. Sekulović and Hana Fatkić [12].

We first show that (1.7) holds, not only for the diameter, but also for the geometric diameter $\delta_{k}(A)$ of order $k$ which is defined for any positive integer $k \geq 2$ and any $A \subseteq X$ by (1.3). Next we show that essential diameter in (1.8) can be replaced by the essential geometric diameter ess $\delta_{k}(A)$ of order $k$ which is defined for any positive integer $k \geq 2$ and any $A \subseteq X$ by (1.5). But here the requirement that transformation $T$ be measurabilitypreserving is necessary because the essential geometric diameter is defined only for measurable sets. All of these results have some importance, not only in Mathematics, but also in Statistical Mechanics.

We introduce the following definitions.

Definition 2.1. A strongly mixing transformation on a finite measure space $(X, \mathscr{A}, \mu)$ with the property $T(\mathscr{A}) \subseteq \mathscr{A}$ is called a measurability-preserving strongly mixing transformation (with respect to invariant measure $\mu$ ).

Note that measurability-preserving strongly mixing transformations on a finite measure space are generalizations of the invertible strongly mixing transformations (they are not necessarily one-to-one).

Definition 2.2. Suppose that $(X, \mathscr{A}, \mu)$ is a finite measure space and $\Phi:=$ $(X, \mathscr{A}, \mu, T)$ is an abstract dynamical system. Then $\Phi$ is called measurability-preserving strongly mixing if $\Phi$ is strongly mixing and $T(\mathscr{A}) \subseteq \mathscr{A}$.

Lemma 2.1. Let $(X, \mathscr{A}, \mu)$ be a finite measure space. Let $T$ be a transformation on $X$ that is strongly mixing with respect to $\mu$ and suppose that $B_{1}, B_{2}, \ldots, B_{k}(k \in \mathbf{N})$ are $\mu$-measurable subsets of $X$. If $\mu(A)>0$ and $\mu\left(B_{i}\right)>0(i=1, \ldots, k)$, then there is a positive integer $n_{0}=n_{0}(k)$ such that

$$
T^{n}(A) \cap B_{i} \neq \varnothing
$$

for $i=1,2, \ldots, k$ and all $n>n_{0}$.

Proof. Since $T$ is strongly mixing and $\mu(A) \mu\left(B_{i}\right)>0$ for all $i \in\{1, \ldots, k\}$, it follows that there is a positive integer $n_{0}=n_{0}(k)$ such that

$$
\mu\left(A \cap T^{-n}\left(B_{i}\right)\right)>0
$$

for $i=1,2, \ldots, k$ and all $n>n_{0}$, whence

$$
A \cap T^{-n}\left(B_{i}\right) \neq \varnothing
$$


for $i=1,2, \ldots, k$ and all $n>n_{0}$. Since

$$
T^{-n}\left(T^{n}(A) \cap B_{i}\right)=\left(T^{-n}\left(T^{n} A\right)\right) \cap T^{-n}\left(B_{i}\right) \supseteq A \cap T^{-n} B_{i}
$$

for all $i=1,2, \ldots, k$ and every $n$ in $N, n>n_{0}$, it follows, by (2.2), that $T^{-n}\left(T^{n}(A) \cap B_{i}\right) \neq \varnothing$ and therefore (2.1) holds and the proof of Lemma 2.1 is complete.

An induction argument yields the following:

Lemma 2.2. If $0 \leq a_{k} \leq a$, for $k=1,2, \ldots, \lambda$ and $b>0$, then

$$
\prod_{k=1}^{\lambda}\left(a_{k}+b\right)-\prod_{k=1}^{\lambda} a_{k} \leq(a+b)^{\lambda}-a^{\lambda} .
$$

Theorem 2.1. Let $(X, d)$ be a metric space, let $\mathscr{A}$ be a $\sigma$-algebra of subsets of $X$ and $\mu$ a finite measure on $\mathscr{A}$. Suppose further that every open ball in $(X, d)$ is $\mu$-measurable and has positive measure. Let $T$ be a transformation on $X$ that is strongly mixing with respect to $\mu$. Then, for any $\mu$-measurable subset $A$ of $X$ of positive measure, and any positive integer $k \geq 2$,

$$
\lim _{n \rightarrow \infty} \delta_{k}\left(T^{n}(A)\right)=\delta_{k}(X),
$$

where $\delta_{k}$ is the geometric diameter of order $k$ given by (1.3).

Proof. Throughout this proof we will denote $\delta_{2}(X)$, the (ordinary) diameter of $X$, by $\delta$.

Let $k$ be an arbitrary positive integer $>1$. Since $\delta_{k}$ is a monotone nondecreasing set function (see [14]), it is clear that (2.5) holds when $\delta_{k}(X)=0$.

Suppose next that $0<\delta_{k}(X)<+\infty$, whence also $0<\delta<+\infty$, and let $\varepsilon>0$ be given. Then, by (1.3), there exist points $x_{1}, x_{2}, \ldots, x_{k}$ in $X$ such that

$$
\left(\sqrt[k]{2} \sqrt{\prod_{1 \leq i<j \leq k} d\left(x_{i}, x_{j}\right)}>\delta_{k}(X)-\frac{\varepsilon}{2} .\right.
$$

Let $m(=m(k, \varepsilon))$ be a positive integer such that

$$
\delta \cdot\left[\left(1+\frac{1}{m \delta}\right)^{\left(\begin{array}{c}
k \\
2
\end{array}\right)}-1\right]^{\frac{2}{k(k-1)}}<\frac{\varepsilon}{2}
$$

and let $B_{1}, B_{2}, \ldots, B_{k}$ be open balls of radius $1 / 2 m$ centered at $x_{1}, x_{2}, \ldots$, $x_{k}$, respectively.

Next, since $T: X \rightarrow X$ is strongly mixing with respect to $\mu$ and since $\mu(A)>0$ and $\mu\left(B_{i}\right)>0$ for all $i=1,2, \ldots, k$, it follows from Lemma 2.1 that there is a positive integer $n_{0}=n_{0}(k, m)$ such that $T^{n}(A) \cap B_{i} \neq \varnothing$ for $i=1,2, \ldots, k$ and all $n>n_{0}$. 
Given $n \geq n_{0}$, for each $i=1,2, \ldots, k$, choose a point $y_{i}$ in $T^{n}(A) \cap B_{i}$. Then, for all $i, j=1, \ldots, k$,

$$
d\left(x_{i}, x_{j}\right) \leq d\left(x_{i}, y_{i}\right)+d\left(y_{i}, y_{j}\right)+d\left(y_{j}, x_{j}\right)<d\left(y_{i}, y_{j}\right)+\frac{1}{m},
$$

whence, using (2.6), we have

$$
\delta_{k}(X)-\frac{\varepsilon}{2}<\left(\sqrt[k]{2} \sqrt{\prod_{1 \leq i<j \leq k} d\left(x_{i}, x_{j}\right)}<\sqrt[k]{\sum_{1 \leq i<j \leq k}\left[d\left(y_{i}, y_{j}\right)+\frac{1}{m}\right]} .\right.
$$

Since $\prod_{1 \leq i<j \leq k} d\left(y_{i}, y_{j}\right) \leq\left(\delta_{k}\left(T^{n}(A)\right)\right)^{\frac{k(k-1)}{2}}$ and $d\left(y_{i}, y_{j}\right) \leq \delta$ for all $i, j=1,2, \ldots, k$, using Lemma 2.2 , with $a_{i j}=d\left(y_{i}, y_{j}\right)$ and $b=\frac{1}{m}$, we obtain

$$
\begin{aligned}
& \prod_{1 \leq i<j \leq k}\left[d\left(y_{i}, y_{j}\right)+\frac{1}{m}\right]=\prod_{1 \leq i<j \leq k} d\left(y_{i}, y_{j}\right)+\left\{\prod_{1 \leq i<j \leq k}\left[d\left(y_{i}, y_{j}\right)+\frac{1}{m}\right]\right. \\
&\left.-\prod_{1 \leq i<j \leq k} d\left(y_{i}, y_{j}\right)\right\} \\
& \leq\left[\delta_{k}\left(T^{n}(A)\right)\right]^{\left(\begin{array}{c}
k \\
2
\end{array}\right)}+\left\{\left(\delta+\frac{1}{m}\right)^{\left(\begin{array}{c}
k \\
2
\end{array}\right)}-\delta^{\left.\left(\begin{array}{c}
k \\
2
\end{array}\right)\right\} .}\right.
\end{aligned}
$$

Using the fact that

$$
\sqrt[n]{a+b} \leq \sqrt[n]{a}+\sqrt[n]{b}
$$

for all $a \geq 0, b \geq 0$, and $n=1,2, \ldots$, it follows that

$$
\delta_{k}(X)-\frac{\varepsilon}{2} \leq \delta_{k}\left(T^{n}(A)\right)+\delta \cdot\left[\left(1+\frac{1}{m \delta}\right)^{\left(\begin{array}{c}
k \\
2
\end{array}\right)}-1\right]^{\frac{2}{k(k-1)}} .
$$

Hence, by (2.7),

$$
\delta_{k}\left(T^{n}(A)\right)>\delta_{k}(X)-\varepsilon,
$$

for every $n \geq N$, and every $\varepsilon>0$, whence

$$
\lim _{n \rightarrow \infty} \inf \delta_{k}\left(T^{n}(A)\right) \geq \delta_{k}(X) .
$$

But, clearly, $\lim _{n \rightarrow \infty} \sup \delta_{k}\left(T^{n}(A)\right) \leq \delta_{k}(X)$ whence we obtain (2.5).

Finally, the case $\delta_{k}(X)=+\infty$ can be treated by choosing for each positive integer $r$ a $k$-tuple of points $x_{1}, x_{2}, \ldots, x_{k}$ in $X$ such that

$$
\left(\sqrt[k]{2} \sqrt[2]{\prod_{1 \leq i<j \leq k} d\left(x_{i}, x_{j}\right)}>r\right.
$$

and then repeating the previous argument. This completes the proof of Theorem 2.1. 
Corollary 2.1. Under the hypotheses of Theorem 2.1, for any $\mu$-measurable subset $A$ of $X$ of positive measure,

$$
\lim _{k \rightarrow \infty}\left(\lim _{n \rightarrow \infty} \delta_{k}\left(T^{n}(A)\right)\right)=\tau(X)
$$

where $\delta_{k}$ is the geometric diameter of order $k$ given by (1.3), and $\tau$ is the (geometric) transfinite diameter given by (1.4).

Proof. The property (2.11) follows immediately from (2.5) and the fact that $\lim \_n \rightarrow \infty \delta_{k}(X)=\tau(X)$.

Theorem 2.2. Let $(X, d)$ be a metric space and $(X, \mathscr{A}, \mu)$ a finite measure space with the property that every open ball in $(X, d)$ is $\mu$-measurable and has positive measure. Let $(X, \mathscr{A}, \mu, T)$ be a measurability-preserving strongly mixing dynamical system with discrete time. Then, for any $\mu$-measurable subset $A$ of $X$ of positive measure, and any positive integer $k \geq 2$,

$$
\lim _{n \rightarrow \infty} \text { ess } \delta_{k}\left(T^{n}(A)\right)=\delta_{k}(X),
$$

where $\delta_{k}$ is the geometric diameter of order $k$ given by (5), and ess $\delta_{k}$ is the essential geometric diameter of order $k$ which is defined for any any positive integer $k \geq 2$ and any $\mu$-measurable subset $A$ of $X$ by (1.4).

Proof. Note that the hypotheses of Theorem 2.2 are such that $\delta_{k}(X)=$ ess $\delta_{k}(X)$ for any positive integer $k \geq 2$. Let $k$ be an arbitrary positive integer $\geq 2$. Since ess $\delta_{k}$ is a monotone nondecreasing set function it is clear that (2.12) holds when $\delta_{k}(X)=0$. To obtain the result it suffices to prove that (2.12) holds for $0<\delta_{k}(X)<+\infty$.

Suppose next that $0<\delta_{k}(X)<+\infty$, whence also $0<\delta<+\infty$, and let $\varepsilon>0$ be given. Then, by (1.3), there exist points tuple of points $x_{1}, x_{2}, \ldots, x_{k}$ in $X$ such that

$$
(\sqrt[k]{2}) \sqrt{\prod_{1 \leq i<j \leq k} d\left(x_{i}, x_{j}\right)}>\delta_{k}(X)-\frac{\varepsilon}{2} .
$$

Let $m(=m(k, \varepsilon))$ be a positive integer such that

$$
\delta \cdot\left[\left(1+\frac{1}{m \delta}\right)^{\left(\begin{array}{c}
k \\
2
\end{array}\right)}-1\right]^{\frac{2}{k(k-1)}}<\frac{\varepsilon}{2},
$$

and let $B_{1}, B_{2}, \ldots, B_{k}$ be disjoint open balls of radius $1 / 2 m$ centered at $x_{1}, x_{2}, \ldots, x_{k}$, respectively.

Since $T: X \rightarrow X$ is measurability-preserving strongly mixing transformations with respect to $\mu$, it follows from (1.6) that there is a positive integer $N=N(k, m)$ such that

$$
\mu\left(T^{n}(A) \cap B_{i}\right)>0
$$


for $i=1,2, \ldots, k$ and all $n \geq N$. Let $n(n \geq N)$ be given.

Let

$$
B=T^{n}(A) \cap\left(\bigcup_{i=1}^{k} B_{i}\right) .
$$

For every measurable set $C \subseteq B$ such that $\mu(C)=\mu(B)$ we have $\mu(C \cap$ $\left.B_{i}\right)>0$ for $i=1,2, \ldots, k$, whence $C \cap B_{i} \neq \varnothing$ for $i=1,2, \ldots, k$, and, arguing as in the proof of Theorem 2.1, we obtain

$$
\delta_{k}(C)>\delta_{k}(X)-\varepsilon .
$$

It follows that

ess $\delta_{k}\left(T^{n}(A)\right) \geq \operatorname{ess} \delta_{k}(B)=\inf \left\{\delta_{k}(C): C \subseteq B, \mu(C)=\mu(B)\right\} \geq \delta_{k}(X)-\varepsilon$ for every $\varepsilon>0$, and for all positive integers $n \geq N$, whence

$$
\lim _{n \rightarrow \infty} \inf \left[\operatorname{ess} \delta_{k}\left(T^{n}(A)\right)\right] \geq \delta_{k}(X) .
$$

But, clearly,

$$
\lim _{n \rightarrow \infty} \sup \left[\operatorname{ess} \delta_{k}\left(T^{n}(A)\right)\right] \leq \delta_{k}(X)
$$

for every positive integer $n$, whence we obtain (2.12).

This proves Theorem 2.2.

\section{Final Comments, Conjectures, and Conclusions}

There is considerable evidence (see the proof of Theorem 2.2) to support a conjecture that our results (for strongly mixing dynamical systems with discrete time) which are contained in Theorems 2.1 and 2.2 can be extended to strongly mixing dynamical systems with continuous time (see $[3$, pp. 626]).

The ordinary diameter of a set need not be a good measure of its size and shape. A better measure is furnished by the geometric (of order $k>2$, transfinite) diameters (see [19, pp. 181-190 and (in Dover Edition) pp. 295297] and also [6], [7], [13], [14] and [17-20]).

B. Schweizer and A. Sklar have been concluded that comparison of (11. 6.1) with (11.3.5) in [19] and the proof of the Rice's Theorem 1.2 quickly leads to the following conjecture [19, Problem 11.6.5]:

Problem 3.1. (Schweizer and Sklar, 1983) Does Theorem 1.2. remain valid when "diameter" is replaced by "transfinite diameter"?

Note that our Theorem 2.1 in this paper is a first step toward the resolution of the above Problem 3.1. Also note that this problem could be formulated in a more general setting, i.e., not only for the compact set, but also for any set (with positive measure ) in a general metric space. 
The (geometric) transfinite diameter of a compact subset $\mathrm{A}$ of a metric space is closely related, and often equal, to the the capacity, and also to the Čebyšev constant. By the Fundamental Theorem of Classical Potential Theory, we have that, for any compact set $A$ in the complex plane,

$$
\operatorname{cap}(A)=\tau(A)=\text { čeb }(A)
$$

(see, e. g., [14] and [18]]). Thus we may also pose the following conjecture (open problem).

Problem 3.2. Does Rice's Theorem 1.2 remain valid when "diameter" is replaced by "Čebyšev constant"?

Acknowledgement. The authors are very grateful to the referee for his comments which improved the presentation of this paper.

\section{REFERENCES}

[1] P. Billingsley, Ergodic Theory and Information, John Wiley \& Sons, Inc., New YorkLondon-Sydney, 1965.

[2] S. Cerbelli, M. Giona, A. Adrover, M. M. Alvarez, and F. J. Muzzio, Invariant properties of a class of exactly solvable mixing transformations - a measure-theoretical approach to model the evolution of material lines advected by chaotic flows, Chaos Solitons Fractals, 11 (4) (2000), 607 - 630.

[3] I. P. Cornfeld, S. V. Fomin, and YA. G. Sinai, Ergodic Theory, Springer Verlag, NewYork-Heidelberg-Berlin, 1982.

[4] T. Erber, B. Schweizer, and A. Sklar, Mixing transformations on metric Spaces, Comm. Math. Phys., 29 (1973), 311-317.

[5] T. Erber and A. Sklar, Macroscopic Irreversibility as a Manifestation of Micro Instabilities, in: Modern Developments in Thermodynamics (B. Gal - Or, ed.), Israel Universities Press and J. Wiley and Sons, Jerusalem-New York, 1974, pp. 281-301.

[6] H. Fatkić, Note on weakly mixing transformations, Aequationes Math., 43 (1992), $38-44$.

[7] H. Fatkić, On probabilistic metric spaces and ergodic transformations, Ph. D. dissertation, Univ. Sarajevo, 2000, 170 pp; Rad. Mat., 10 (2) (2001), 261-263.

[8] H. Fatkić, Characterizations of measurability-preserving ergodic transformations, Sarajevo J. Math., 1 (13) (2005), 49-58.

[9] H. Fatkić, On measurability-preserving weakly mixing transformations, The First Bosnian-Herzegovinian Mathematical Conference, The Mathematics Department of the Faculty of Natural Sciences and Mathematics of the University of Sarajevo , July 1, 2006; in: Resume of the Bosnian-Herzegovinian mathematical conference, Sarajevo J. Math., 2 (15) (2006), 259-260.

[10] H. Fatkić, Measurability-preserving weakly mixing transformations, Sarajevo J. Math., 2 (15) (2006), 159-172.

[11] H. Fatkić and S. Sekulović, On ergodic transformations on metric spaces, means by Kolmogorov and Nagumo and means by M. Bajraktarević, The Second BosnianHerzegovinian Mathematical Conference, The Mathematics Department of the Faculty of Natural Sciences and Mathematics of the University of Sarajevo, June 23, 
2007; in: Resume of the Bosnian-Herzegovinian mathematical conference, Sarajevo J. Math., 3 (16) (2007), 277-278.

[12] H. Fatkić, S. Sekulović, and Hana Fatkić, Further results on the ergodic transformations that are both strongly mixing and measurability preserving, The sixth BosnianHerzegovinian Mathematical Conference, International University of Sarajevo, June 17, 2011, Sarajevo, B\&H; in: Resume of the Bosnian-Herzegovinian mathematical conference, Sarajevo J. Math., 7 (20) (2011), 310-311.

[13] M. Grandcolas, Regular polygons and transfinite diameter, Bull. Austral. Math. Soc., $62(2000), 67-74$.

[14] E. Hille, Topics in Classical Analysis, in: Lectures on Modern Mathematics (T. L. Saaty, ed.), Vol. 3, Wiley, New York, 1965, pp. 1-57.

[15] A. Kolesárová, R. Mesiar, and C. Sempi, Measure-preserving transformations, copuloe and compatibility, Mediterr. J. Math., 5 (3) (2008), 325-339.

[16] Y. Li and J. Guo, Asymptotic normality of wavelet estimator for strong mixing errors, J. Korean Statist. Soc., 38 (4) (2009), 383-390.

[17] R. E. Rice, On mixing transformations, Aequationes Math., 17 (1978), 104-108.

[18] E. B. Saff, Logarithmic potential theory with applications to approximation theory, Surv. Approx. Theory, 5 (2010), 165-200.

[19] B. Schweizer and A. Sklar, Probabilistic Metric Spaces, North-Holland Ser. Probab. Appl. Math., North-Holland, New York, 1983; second edition, Dover, Mineola, NY, 2005.

[20] C. Sempi, On weakly mixing transformations on metric spaces, Rad. Mat., 1 (1985), $3-7$.

[21] S. V. Tikhonov, Homogeneous spectrum and mixing transformations, (Russian) Dokl. Akad. Nauk , 436 (4) (2011), 448-451; translation in Dokl. Math., 83 (2011), 80-83.

[22] P. Walters, An Introduction to Ergodic Theory, (GTM, Vol. 79), Springer-Verlag, New York-Heidelberg-Berlin, 1982.

[23] P. Walters, Regularity conditions and Bernoulli properties of equilibrium states and g-measures, J. London Math. Soc., II. Ser., 71 (2) (2005), 379-396.

(Received: August 19, 2012)

(Revised: September 29, 2012)
Department of Mathematics

Faculty of Electrical Engineering

University of Sarajevo

Zmaja od Bosne bb (Campus)

Sarajevo, 71000, Bosnia and Herzegovina

E-mails: hfatkic@etf.unsa.ba mehmed89@hotmail.com 